\title{
Redeveloping Drugs Based on Existing Patents
}

\section{Zhao $X Y^{1 *}$, Diao TX ${ }^{1}$ and Zeng $\mathbf{Y J}^{2}$}

${ }^{1}$ Institute of Health Service and Medical Information, Beijing, China

${ }^{2}$ Beijing University of Technology, 100 Pingleyuan, Chaoyang District, Beijing 100022, China

\begin{abstract}
With the advent of the era of knowledge economy and China's join into WTO, the Problem of intellectual property rights of drug has become increasingly important to Pharmaceutical industry and even to all society. The Patent Protection is the most important part of intellectual property right of drug, which does not only act as a safe guard and stimulator to drug development, but also is a Powerful tool for drug development Providing comprehensive technology and law information. The paper analyzed some Pharmaceutical companies' strategies to develop new drug with Patent.
\end{abstract}

An investigation of the U.S. Federal Trade Commission shows that, the pharmaceutical industry is more dependent on patents than any other industries; the industry prevents free competition, compensate R\&D investment, and learn about new technical breakthroughs through patents. Chinese researchers have no strong awareness of using patent literatures, often neglecting acquisition and analysis of patent information. Therefore, the author summarized and analyzed current methods of how foreign pharmaceutical companies use patent information for drug development, to provide Chinese pharmaceutical companies with references on fully using patent resources to develop drugs.

\section{Development of Me-Too Compound Patent}

The most common strategy adopted by many large-scale Western pharmaceutical companies is to modify a patented compound to produce a new compound and apply for patent for it. But the premise is no violation of the original patent. "me-too" drug normally refers to a new kind of molecular entity [1]. It has similar chemical structure or the same pharmacological effect with a certain drug that is already on the market. And it enjoys some advantages in treatment. . Sometimes, this "me-too" strategy is also called fast follower strategy. Pharmaceutical companies need to follow closely the newly disclosed drug patents, especially the drugs with new mechanism of action and chemical structure that have not come into the market yet. The ideas and technologies of these drug patents are often groundbreaking. But the drug development is not mature enough, leaving some potential for modification [2]. The transformation of proton pump inhibitor (PPI) by Japanese company Takeda is a successful case of "me-too" strategy. The first PPI, called omeprazole, was developed by Astra and came into the market in 1987. Then the second PPI, lansoprazole, developed by Takeda began to be sold in 1992. Both PPIs applied for the patent. Structural comparison indicates that lansoprazole is the analogue of omeprazole. That is to say, lansoprazole is a "me-too" drug.

In terms of the scope of patent protection, the two PPIs are nearly identical except for a fluorine-substituted alkyl group. Lansoprazole is the product of structural modification and innovation on omeprazole, which fully considered the protection scope of omeprazole and hence has no infringement of its patent right. Lansoprazole has the similar mechanism of action with omeprazole. But through structural innovation, lansoprazole's lipophilicity has been improved, and the bioavailability increased by $30 \%$ compared with omeprazole. Besides, the former's antibacterial activity against Helicobacter pylori is about 4 times that of the latter. Hence lansoprazole can move more quickly through wall cells to play its efficacy, and rapidly relieve pain, heartburn and other symptoms [3].

The conditions required for "me-too" drug development have been summarized as follows: (1) The pharmaceutical companies should have their own R\&D institute and staff, particularly stronger medicinal chemistry and pharmacology teams. (2) A well-developed information department is required. The talents who have good knowledge in both pharmacy and patent law need to be cultured. Thus, the companies are able to track rapidly development and patent information of groundbreaking new drugs. (3) Patent literatures concerning the improvable patent compound should be collected. In the meantime, the patent literature database should be established for internal R\&D staff. Thus, the companies can prevent the leakage of technology secret during the retrieval in external databases, while ensuring the full utilization of domestic and foreign patent literatures. (4) Much attention should be paid to the combination of patent literature investigation and market research. The thematic investigation of patent literatures should be carried out to learn the technical level, development status and trend of related fields. Meanwhile, market research should also be conducted regularly to master market demands [4].

\section{Development of Peripheral Patent}

Another common strategy of utilizing patents is to look for patent breach or redevelop the patents. By retrieving related literatures of patented drugs, you can find deficiencies in existing patents and transform them to form peripheral patents to achieve cross-licensing. Alternatively, you can redevelop the drugs with expired patents to form new patented drugs.

\section{Separating isomers of single component}

Currently, chiral drugs have become one of the new directions of international drug research. The rapidly growing market demands stimulate research and development of chiral drugs. Single-enantiomer drugs grow at a rate of more than $20 \%$ each year in the world market. By revealing the pharmacological, toxicological and metabolic characteristics of the enantiomer's single-component isomers, as well as their different clinical performances, the new drugs with stronger receptor affinity, shorter onset time, and smaller side effects can be developed.

*Corresponding author: Zhao XY, Institute of Health Service and Medical Information, Beijing, China, E-mail: yjzeng@bjut.edu.cn

Received March 02, 2016; Accepted August 02, 2016; Published August 09 2016

Citation: Zhao XY, Diao TX, Zeng YJ (2016) Redeveloping Drugs Based on Existing Patents. Primary Health Care 6: 233. doi:10.4172/2167-1079.1000233

Copyright: @ 2016 Zhao XY, et al. This is an open-access article distributed under the terms of the Creative Commons Attribution License, which permits unrestricted use, distribution, and reproduction in any medium, provided the original author and source are credited. 
A successful case is presented as follows. The SePracor company (Massachusetts, the U.S.), founded in 1984, focuses on developing isomer separation techniques and applying for patent protection for those large pharmaceutical companies. Since 1989, the development direction of this company converted from developing isomer separation techniques to redeveloping the drugs of other companies on the market and applying for patent protection for the redeveloped drugs. For example, SePracor has successfully obtained the patent of fluoxetine, which is the single optical isomer (R) of the antidepressant racemic fluoxetine developed by Eli Lilly and Company and put it on the market in 2001. While developing the S-enantiomer of enantiomer as a non-steroidal anti-inflammatory drug (NSAID), SePracor is also developing the R-enantiomer as a toothpaste additive for prevention of periodontitis. It provides an example for the strategy proposed in this section [5].

With the development of chiral drugs, there are fewer changes of developing single-component isomers. The primary reason is that since the early 1990s, the U.S. Food and Drug Administration (FDA) has begun to encourage large pharmaceutical companies to develop single-isomer drugs to replace racemates. There are several methods of developing single-component isomers: (1) The separation of single-component isomers should be performed on those effective drugs with optical activity and obvious adverse reactions. (2) Patent retrieval is performed on the candidate's drugs. According to the patent information, the drug containing a single component of optical isomers that is not protected by patent will be selected for separation. (3) According to type of selected drugs, the chiral separation methods of their similar compounds are searched as reference. Generally, chiral drugs are synthesized through chemical synthesis method and biological catalytic reaction. Besides, the direct chiral drug separation methods can also be employed, including resolution by direct crystallization, chemical resolution, column chromatography, and kinetic resolution.

\section{Obtaining patents of human metabolite}

Using human metabolite patent to extend the patent protection of a certain drug is a new strategy of patent protection. Bristol-Myers Squibb (BMS) Company successfully applied this strategy. The antianxiety drug buspirone hydrochloride (BusPar) is one of the important products of BMS. BMS estimated that once the patent of BusPar expires and generic drug flood into the market, the BusPar sales would reduce by $80 \%$. To address this issue, BMS analyzed the metabolites of BusPar in human body, and identified the strong anxiolytic activity of a metabolite, 6-hydroxy buspirone. Accordingly, BMS applied for a new patent (US 6150365), achieving the purpose of extending the original patent protection period [6]. If this metabolite is successfully identified by pharmaceutical companies other than BMS, they can also apply for the dependent patent of BMS for it. The dependent patent provides an opportunity to extend the patent protection period of original compound. Thus the owner of dependent patent, together with that of original compound patent, can achieve the mutual benefit by patent cross-licensing. The applicability and methods of metabolite development are introduced as follows: (1) The development of metabolites is more suitable for the original R\&S company of a certain drug. Such company must provide pharmacokinetic experimental data before applying for new drug. Due to the advantage in data, they generally deduce relevant metabolites and carry out pharmacodynamic studies earlier. (2) Another method is to collect human metabolite data of various drugs and then entrust a specialized agency to carry out pharmacodynamic experiments. It is similar to small-scale drug screening. It is noteworthy that the patent literatures of original drug must be analyzed carefully to determine whether its metabolites are within the scope of protection, so as to avoid literal infringement and infringement under the doctrine of equivalents.

\section{Changing administration route or crystal form of drugs}

By retrieval of patent literatures, one can learn all formulations covered by the patent of a drug, and identify the formulation available for development to improve drug efficacy, reduce toxicity or make it more convenient for use. Through the application of this strategy, the Swiss Alcon company has successfully applied for a topical suspension preparation containing ciprofloxacin and dexamethasone. Although containing nonionic polymer, nonionic surfactant and ionic reinforcing agent, the formulation has stable physical properties. It is easy to be applied topically to the eye, ear or nose [7].

The development of new drug formulations has a relatively low cost and can be outsourced. According to statistics, the development of a new formulation of existing drugs requires only 50 million dollars, while the maximum sales may reach up to 500 to 700 million dollars with the failure rate of only $20 \%$.

\section{Changing production method-related patents of others or synthesizing new intermediates}

Generally, it is not difficult to produce a patented drug by bypassing its patented method. Modifying drug production methods is quite common in pharmaceutical companies. On the basis of in-depth research on existing patents, pharmaceutical companies are able to form more advantageous new production methods through continuous explorations in production.

For example, the U.S. Lake Pharmaceutical and Chemical Company have successfully obtained a patent for the preparation method of a highpurity HMG-CoA reductase inhibitor. With the use of displacement chromatography, this invention can produce HMG-coA reductase inhibitor with high purity, high yield, and low cost, which is suitable for ecological balance. The accumulation of impurities in low-purity drugs may cause many adverse reactions in treatment.

When you develop a new synthetic route, refinement or purification method, or find a new intermediate, you can apply for the patent for them. The new methods generally have one of following features: (1) Easy to operate, easy for industrial production, and lower cost; (2) improving the purity and stability of products; (3) Improving efficacy and reducing side effects; (4) Reducing environmental pollution and energy consumption; (5) Improving product quality; (6) Improving physical properties (e.g. prolonging storage life, enhancing product stability); (7) Improving the controllability of quality standards.

\section{Developing new use of existing drugs}

Developers always apply for patent as soon as possible to take the initiative in competition. It is impossible for them to conduct comprehensive screening, and toxicological and pharmacological studies for the compound to be patented. Therefore, pharmacological research of not disclosed or studied therapeutic use can be performed for the compounds with disclosed structural formula to find new therapeutic use. Foreign pharmaceutical companies pay great attention to the development of new uses for drug. They often continue to apply for patent for new uses of a compound for many years after it has been patented.

Finasteride was initially a prescription drug used to treat prostatic hyperplasia as it can block the enzyme catalyzing the conversion from testosterone to dihydrotestosterone. After the redevelopment of Merck 
Company, low-dose finasteride can be used for the treatment of typical male baldness. In 1998, FDA approved it as a therapeutic agent for alopecia $[8,9]$.

Pharmaceutical companies can assign specific staff to collect adverse reaction data of drugs during animal experiments and clinical trials, and establish corresponding databases and screening methods. It will provide ideas for the exploration of new uses of a drug. The discovery of new therapeutic uses has a large contingency. Its successful cases are very few in comparison to the development of new production methods and new formulations.

\section{Developing new crystal forms or amorphous state}

The same element or compound may generate completely different structures, morphologies, and physical properties under different condition. This phenomenon is referred to as polymorphism. The physical and chemical properties and bioavailability will change with the crystal form to a varying extent [10].

According to the statistics of tablet samples in United States Pharmacopoeia (20th Edition), about $40 \%$ of the drugs show the polymorphism. A growing number of patent applications also show that exploring suitable crystal forms to improve the drug solubility and bioavailability has become a very important route of drug redevelopment.

For example, among 29 Chinese patent applications related to omeprazole, three claimed protection for different new crystal forms of omeprazole [11].

To develop a new crystal form, the first step is to screen the polymorphic compounds with similar properties as the given drug. Then through patent retrieval, a patent database of crystal forms is established, as technical reference for crystal form development.

\section{Developing new salts, aceta and new thermodynamically stable hydrates and complexes}

In order to solve many problems in stability and bioavailability of certain drugs, specific molecules need to be added to react with the drugs, to form stable compounds or improve their properties. Specifically, the drugs with similar pharmacological properties to the given drug, especially those with the same defects, should be identified first. Then the related patents of the identified drugs are searched and analyzed to find developing ideas for improving pharmaceutical properties.

The Swiss Sandoz stock company has applied for a patent related to new salt and polymorphs of desloratadine hemifumarate, which provides a method for preparing new polymorphs of desloratadine hemifumarate. Characteristics of these polymorphs are as follows: The salts of desloratadine in water and protonic organic solvent show much higher solubility than the desloratadine itself [12].

\section{Developing drug combination or compound to treat specific diseases}

The German company Bayer has successfully applied for nonirritating, non-sensitizing and non-ototoxic otic antimicrobial compositions. The compositions are water-based, and comprise (1) an effective dose of ciprofloxacin (as an antibacterial ingredient) or its pharmaceutically acceptable salt; (2) an effective dose of hydrocortisone (as an anti-inflammatory ingredient) or its pharmaceutically acceptable salt; and (3) an effective amount of polyvinyl alcohol with the degree of hydrolysis being at least $85 \%$ to suspend hydrocortisone in solution [13]. The primary problem faced by the above development strategies of peripheral patent is to correctly understand and handle the legal status of existing drug patents. The company first developing a trademarked drug possesses the composition patent for the compound, that is, the exclusive rights to develop, manufacture and sell the compound. The companies that developed the peripheral patents of a drug cannot sell and produce the drug before its patent expires, unless they get permission of the original research company. Therefore, for trademarked drugs protected by the material composition patent, many companies prefer to seek external permission, namely, cross-licensing with the original research company.

\section{Current Patent Utilization Status of Chinese Pharmaceutical Companies}

Chinese pharmaceutical companies have made some achievements on redeveloping drugs, but few of them are able to take advantage of patent information to guide drug development. R\&D departments of these companies often depend too much on scientific and technical journals but neglect patent literatures. In fact, in addition to the methods described above, different companies can summarize more redevelopment methods suitable for themselves from patent literatures. Pharmaceutical companies should pay attention to the patent knowledge training and patent awareness cultivation of their drug R\&D staff [14]. It is essential to culture the comprehensive talents that are proficient in both pharmacy and patent knowledge. Thus, the seeking of entry point of R\&D and developing methods of reference value from a large number of patent literatures will become easier. Furthermore, the companies should establish specialized patent information sector, assign specialized staff to collect patent information, and construct internal patent database.

\section{References}

1. Fabry B, Ernst H, Langholz J, Koster M (2006) Patent portfolio analysis as a useful tool for identifying R\&D and business opportunities-an empirical application in the nutrition and health industry. World Patent Information 28:215-225.

2. DiMasi JA, Paquette C (2004) The economics of follow-on drug research and development: trends in entry rates and the timing of development Pharmacoeconomics 22:1-14.

3. Liu $Y$ (2001) Recent progress in proton pump inhibitors. Chinese Journal of New Drugs 10: 161-164.

4. Yuan Z, Liu C, Wang Y (2001) New drug development and patent literature utilization. Chinese Journal of New Drugs 10: 724-726.

5. Wang $P$ (1999) New strategy of drug research and development-improved chemical entities. Chinese Journal of New Drugs10: 656.

6. Zhou H (2003) Strategies and techniques of pharmaceutical intellectual property protection. Journal of China Pharmaceutical University 34: 90-92.

7. Swiss Alcon Company (2002) Topical suspension formulations containing ciprofloxacin and dexamethasone. China 00813262.

8. Farmacevtskih LT (2001) Preparation of high-purity HMG-coA reductase inhibitors. China, 99803080.

9. Innovative strategies of drug redevelopment (EB/oL)

10. Hao W, Zhu R (2004) Intensive study of drug crystal form is essential. Science \& Technology Vision 13: 19-553.

11. Wu S, He W (2004) Observation of the patent strategy of drug research and development-from the perspective of paroxetine mesylate. Chinese Pharmaceutical Journal 39: 551-583.

12. Swiss Sandoz Stock Company (2005) Novel salt and polymorphs of desloratadine hemifumarate. China 03818836.

13. Yang L, Wang J, Sun $Y$ (2004) A phase I clinical trial for recombinant human endostatin. Chinese Journal of New Drugs 13: 545-553.

14. German Bayer Company. Non-irritation, non-sensitizing and non-ototoxic otic antibacterial compositions. China 96190570. 